\title{
Metamorphosis of the invasive ascidian Ciona savignyi: environmental variables and chemical exposure
}

Patrick L Cahill, Javier Atalah, Andrew I Selwood, Jeanne M Kuhajek

In this study, the effects of environmental variables on larval metamorphosis of the solitary ascidian Ciona savignyi were investigated in a laboratory setting. The progression of metamorphic changes were tracked under various temperature, photoperiod, substrate, larval density, and vessel size regimes. Metamorphosis was maximised at $18^{\circ} \mathrm{C}, 12: 12 \mathrm{~h}$ subdued light:dark, smooth polystyrene substrate, and 10 larvae $\mathrm{mL}^{-1}$ in a twelve-well tissue culture plate. Eliminating the air-water interface by filling culture vessels to capacity further increased the proportion of metamorphosed larvae; $87 \pm 5 \%$ of larvae completed metamorphosis within 5 days compared to $45 \pm 5 \%$ in control wells. The effects of the reference antifouling compounds polygodial, portimine, oroidin, chlorothalonil, and tolylfluanid on $C$. savignyi were subsequently determined, highlighting (1) the sensitivity of C. savignyi metamorphosis to chemical exposure and (2) the potential to use $C$. savignyi larvae to screen for bioactivity in an optimised laboratory setting. The compounds were bioactive in the low $\mathrm{ng} \mathrm{mL}^{-1}$ to high $\mu \mathrm{g} \mathrm{mL}^{-1}$ range. Polygodial was chosen for additional investigations, where it was shown that mean reductions in the proportions of larvae reaching stage $E$ were highly repeatable both within (repeatability $=14 \pm 9 \%$ ) and between (intermediate precision $=17 \pm 3 \%$ ) independent experiments. An environmental extract had no effect on the larvae but exposing larvae to both the extract and polygodial reduced potency relative to polygodial alone. This change in potency stresses the need for caution when working with complex samples, as is routinely implemented when isolating natural compounds from their biological source. Overall, the outcomes of this study highlight the sensitivity of $C$. savignyi metamorphosis to environmental variations and chemical exposure. 
3 Metamorphosis of the invasive ascidian Ciona savignyi: environmental variables and chemical exposure

Patrick L. Cahill a, Javier Atalah a, Andrew I. Selwood a, \& Jeanne M. Kuhajek a

16 Corresponding author: Patrick Cahill; Cawthron Institute, 98 Halifax St East, Nelson

17 7010, New Zealand; Phone: +643 548 2319; Email: patrick.cahill@cawthron.org.nz 


\section{Abstract}

In this study, the effects of environmental variables on larval metamorphosis of the solitary ascidian Ciona savignyi were investigated in a laboratory setting. The progression of metamorphic changes were tracked under various temperature, photoperiod, substrate, larval density, and vessel size regimes. Metamorphosis was maximised at $18^{\circ} \mathrm{C}, 12: 12 \mathrm{~h}$ subdued light:dark, smooth polystyrene substrate, and 10 larvae $\mathrm{mL}^{-1}$ in a twelve-well tissue culture plate. Eliminating the air-water interface by filling culture vessels to capacity further increased the proportion of metamorphosed larvae; $87 \pm 5 \%$ of larvae completed metamorphosis within 5 days compared to $45 \pm 5 \%$ in control wells. The effects of the reference antifouling compounds polygodial, portimine, oroidin, chlorothalonil, and tolylfluanid on C. savignyi were subsequently determined, highlighting (1) the sensitivity of C. savignyi metamorphosis to chemical exposure and (2) the potential to use $C$. savignyi larvae to screen for bioactivity in an optimised laboratory setting. The compounds were bioactive in the low $\mathrm{ng} \mathrm{mL}^{-1}$ to high $\mu \mathrm{g} \mathrm{mL} \mathrm{L}^{-1}$ range. Polygodial was chosen for additional investigations, where it was shown that mean reductions in the proportions of larvae reaching stage $E$ were highly repeatable both within (repeatability $=14 \pm 9 \%$ ) and between (intermediate precision $=$ $17 \pm 3 \%$ ) independent experiments. An environmental extract had no effect on the larvae but exposing larvae to both the extract and polygodial reduced potency relative to polygodial alone. This change in potency stresses the need for caution when working with complex samples, as is routinely implemented when isolating natural compounds from their biological source. Overall, the outcomes of this study highlight the sensitivity of $C$. savignyi metamorphosis to environmental variations and chemical exposure. 
41

42

43

44

\section{Introduction}

The Pacific transparent ascidian Ciona savignyi Herdman (Cionidae) is a Japanese native with an expanding invasive range that currently includes Argentina, British Columbia, California, New Zealand, Puget Sound, and Spain (Fofonoff et al. 2003, Lambert and Lambert 1998, Smith et al. 2010). Ciona savignyi is recognised as a problematic biofouling organism; it reproduces rapidly in invaded environments and can dominate man-made and natural substrates (e.g., Cohen et al. 1998, Zvyagintsev et al. 2007). This hermaphroditic species can spawn year-round in temperate regions (Nomaguchi et al. 1997; P. Cahill pers obs), with each individual releasing hundreds or thousands of eggs to be fertilised in the water column (Hendrickson et al. 2004, Jiang and Smith 2005).

Any attempt to counteract the invasive tendencies of $C$. savignyi requires an indepth understanding of this species life-history characteristics. A key step in the biofouling process is the transition from free-swimming larva to sessile adult (Pawlik 1992). Larvae must contact a suitable surface upon which to settle, choose to attach, and then undergo a complex series of morphological changes to form established juveniles. Marine larvae typically respond to a range of environmental and con-specific cues, with a high degree of variability in responses between species (Jackson et al. 2002, Rodriguez et al. 1993). Little information is available on the extrinsic factors that stimulate C. savignyi to metamorphose; identifying these cues will facilitate the development of targeted treatment technologies and mitigation techniques. 
In particular, the metamorphic process may be sensitive to exposure to chemical

63

64

65

66

67

68

compounds. It has been shown that $C$. savignyi metamorphosis is inhibited by the natural antifouling agent polygodial (Cahill and Kuhajek 2014), with other natural (e.g., oridin or portimine; Selwood et al. 2013, Tsukamoto et al. 1996) and synthetic (e.g., chlorothalonil and tolyfluanid; Voulvoulis et al. 1999) compounds also likely to be effective against C. savignyi metamorphosis. Screening for effects on C. savignyi metamorphosis has potential to identify targeted lead compounds, including both known compounds and novel natural compounds. In the case of novel natural compounds, initial screening and isolation typically involves working with complex biological extracts, with potential for interactions between constituents of the extract (Colegate and Molyneux 2007). Understanding how environmental factors influence metamorphosis will improve our ability to reliably quantify antimetamorphic effects.

In addition to the interest in $C$. savignyi as a marine invader, this species has been increasingly studied as a model organism for developmental biology (Corbo et al. 2001, Sasakura et al. 2012, Satoh 2003). Ascidians occupy an intriguing evolutionary position as sister clade to the vertebrates (Lemaire 2011, Satoh and Levine 2005, Schubert et al. 2006), meaning they a can afford insights into developmental biology in general. Embryos and larvae of $C$. savignyi can be produced in large numbers in the lab, the latter undergoing a defined progression of metamorphic changes to form established juveniles within 7 days (Cirino et al. 2002, Hendrickson et al. 2004, Kourakis et al. 2010). Many studies have examined the intrinsic determinants driving the progression from egg, to free-swimming larvae, to sessile adult in C. savignyi (e.g., Imai et al. 2002a, Imai et al. 2002b, Kimura et al. 2003). Identifying extrinsic conditions that 
85 stimulate ascidian larvae to settle and metamorphose will also provide a context for 86 these molecular and biochemical investigations (Morse 1990).

87 In this study, the effects of extrinsic environmental factors on C. savignyi 88 metamorphosis were examined in a laboratory setting. Variables investigated included 89 temperature, photoperiod, substrate, larval density, and vessel size. The most 90 susceptible stages of the larval settlement and metamorphosis processes were 91 identified, providing a relevant experimental end-point for investigating the effects of 92 reference antifouling compounds on the larvae. 
93

94

95

96

97

98

99

100

101

102

103

104

105

106

107

108

109

110

111

112

113

114

\section{Material and Methods}

\subsection{Culture and Spawning}

Adult C. savignyi, collected from the underside of pontoons at Nelson Marina, Nelson, New Zealand, were housed in water lily baskets suspended in 10-L glass aquaria for up to three weeks. Aquaria were supplied with $10 \mathrm{~L}$ seawater $\mathrm{h}^{-1}$ as part of a $1000-\mathrm{L}$ recirculating system held at $18 \pm 1^{\circ} \mathrm{C}, 34 \pm 1 \mathrm{psu}$, and $300 \pm 50 \mathrm{mV}$ ORP; constant fullspectrum florescent light prevented premature spawning. Daily, the flow of water to the aquaria was cut off for $3 \mathrm{~h}$ while $C$. savignyi were fed $250 \mathrm{~mL}$ of an $8-9 \times 10^{6}{\text { cells } \mathrm{mL}^{-}}^{-}$ 1 Isochrysis galbana Parke culture. Three gravid individuals with densely packed egg and sperm ducts were spawned according to Cirino et al. (2002). Ventral incisions were made to expose the egg and sperm ducts. The egg duct of each individual was pierced with a Pasteur pipette and the eggs transferred to a glass Petri dish $(90 \mathrm{~mm}$ dia., $68 \mathrm{~mL}$ vol.) filled with $20 \mathrm{~mL}$ of $0.3-\mu \mathrm{m}$ filtered and UV-sterilized seawater (FSW). Sperm were then harvested and transferred to a glass Petri dish (90 mm dia., $68 \mathrm{~mL}$ vol.) containing $50 \mathrm{~mL}$ FSW. Each dish of eggs received eight drops of sperm suspension from each of the two other individuals. After incubating at $18^{\circ} \mathrm{C}$ for $1 \mathrm{~h}$, fertilized eggs were strained through a $20-\mu \mathrm{m}$ sieve, rinsed three times with $25 \mathrm{~mL}$ reconstituted seawater (RSW; 33

$\pm 0.5 \mathrm{psu}$; Red Sea Salt, Red Sea Aquatics, Cheddar, UK), transferred to a glass Petri dish along with $25 \mathrm{~mL} \mathrm{RSW}$, and held at $18^{\circ} \mathrm{C}$ for $18 \mathrm{~h}$ to hatch. Hatched larvae were transferred to conical flasks and diluted with RSW as necessary to yield desired larval densities.

\subsection{Temperature, photoperiod, and substrate}


115 The temperature, photoperiod, and substrate preferences of larvae were assessed in

116 three separate experiments. Firstly, the effects of exposing larvae to $15,18,21$, and

$11725^{\circ} \mathrm{C}$ were evaluated. For the photoperiod experiment, larvae were exposed to the 118 following lighting regimes: 24:0, 12:12, 0:24 $\mathrm{h}$ intense $\left(100 \pm 10 \mu \mathrm{mol} \mathrm{m}^{-2} \mathrm{~s}^{-1}\right)$ or 119 subdued $\left(10 \pm 5 \mu \mathrm{mol} \mathrm{m} \mathrm{m}^{-2} \mathrm{~s}^{-1}\right)$ light:dark. Substrate type (polystyrene, acrylic, or glass) 120 and texture (smooth or rough) were assessed using unlined wells (polystyrene) or wells

121 lined with acrylic discs (35-mm diameter) or glass cover slips (35-mm diameter, Gerhard 122 Menz $\mathrm{GmbH}$, Saarbrücken, GE). Untreated unlined wells, acrylic discs, and glass cover 123 slips comprised smooth treatments. Sandblasted acrylic and sanded (800-grit 124 sandpaper) glass and polystyrene comprised rough treatments; it should be noted that 125 these surface treatments likely resulted in different feature sizes for each of the three 126 substrate types and that the walls of the wells remained untreated in all cases.

All three experiments (temperature, photoperiod, and substrate) were performed 128 in six-well tissue culture plates (Corning ${ }^{\circledR}$ Costar $^{\circledR}$, Corning Inc., Corning, NY; 36 mm dia.; $17.5 \mathrm{~mL}$ vol.) filled with $10-\mathrm{mL}$ aliquots of 2.5 larvae $\mathrm{mL}^{-1} \mathrm{RSW}$. Default parameters were $18^{\circ} \mathrm{C}, 12: 12 \mathrm{~h}$ subdued light:dark, and unlined smooth wells; three replicates were performed in all cases $(n=3)$. Culture waters were renewed with RSW every other day and settled larvae were scored according to metamorphic stage (Figure 1) after 1, 3, 5, and 7 days. Unattached larvae or larvae adhered to the meniscus were not counted. A proxy for daily metamorphic progress was calculated based on the number of larvae 135 counted at each developmental stage as follows:

Metamorphic progress 


\section{2.3. Vessel size, larval density, and fill volume}

138 Vessel size was assessed in conjunction with larval density. Polystyrene Petri dishes

139 (LabServ, Thermo Fisher Scientific, AU; $90 \mathrm{~mm}$ dia.; $68 \mathrm{~mL}$ vol.), six-well plates, and 140 twelve-well plates (Corning ${ }^{\circledR}$ Costar $^{\circledR} ; 23 \mathrm{~mm}$ dia.; $6.5 \mathrm{~mL}$ vol.) were filled with 25-, 10-, 141 or 5-mL aliquots of larval suspension, respectively. Four larval densities $(2.5,5,10$, and 14215 larvae $\mathrm{mL}^{-1}$ ) were tested in each of the three vessel sizes, yielding twelve vessel size $143 \times$ larval density treatments in total $(n=3)$. Vessels were held at $18^{\circ} \mathrm{C}, 12: 12 \mathrm{~h}$ subdued 144 light to dark and culture waters were renewed every other day. After 5 days, the number 145 of larvae that had completed stage $\mathrm{E}$ of metamorphosis were counted (Figure 1).

Fill volume was assessed using twelve-well plates and a larval density of 10 larvae $\mathrm{mL}^{-1}$. Treatment wells were filled to capacity with $7.1 \mathrm{~mL}$ of larval suspension so 148 that the seawater contacted the underside of the lid, eliminating the air-water interface. 149 Controls wells were filled with $5 \mathrm{~mL}$ of larval suspension $(\mathrm{n}=3)$. The temperature, 150 photoperiod, and experimental end-point matched those used for the vessel size and 151 larval density experiments but culture waters were not renewed for the duration of this 152 experiment.

\section{$153 \quad 2.4$ Carrier solvent and reference compounds}

154 The sensitivity of larvae to two common carrier solvents was assessed in twelve-well 155 plates with $7.1 \mathrm{~mL}$ aliquots of 10 larvae $\mathrm{mL}^{-1} \mathrm{RSW}$; held at $18^{\circ} \mathrm{C}, 12: 12 \mathrm{~h}$ subdued light 156 to dark. Concentrations of ethanol and dimethyl sulfoxide (DMSO) evaluated were 0.05, 157 $0.1,0.25,0.5,1,2.5,5,10,20$, and $40 \mu \mathrm{LL}^{-1}$; controls contained no solvent $(\mathrm{n}=3)$.

158 Larvae that had completed stage $\mathrm{E}$ of metamorphosis were counted after 5 days. 
Five reference compounds, polygodial (ENZO Life Sciences, Farmingdale, NY),

160 portimine (Cawthron Natural Compounds, Nelson, NZ), oroidin (ENZO Life Sciences),

161 chlorothalonil (Sigma-Aldrich, St Louis, MO), and tolylfluanid (Sigma-Aldrich), were

162 screened using the protocol outlined for carrier solvent. With the exception of polygodial

$163\left(1,2.5,5,10,15,20,40,80 \mathrm{ng} \mathrm{mL}^{-1}\right)$ and portimine $\left(0.05,0.1,0.5,1,5,10,20 \mathrm{ng} \mathrm{mL}^{-1}\right)$,

164 concentrations tested against the larvae were $0.001,0.005,0.01,1,5$, and $10 \mu \mathrm{gL}^{-1}$.

165 Stock solutions prepared in 20\% (v/v) ethanol (polygodial), RSW (portimine), or 20\%

166 (v/v) DMSO (oroidin, chlorothalonil, tolylfluanid) were added to wells to yield the desired

167 test concentrations; control wells contained solvent only $(n=3)$.

Polygodial treatments $\left(5,10\right.$, and $\left.50 \mathrm{ng} \mathrm{mL}^{-1} ; \mathrm{n}=3\right)$ were subsequently included

in eight independent experiments performed over a 1-year period, allowing repeatability

170 (i.e., intra-run variability) and intermediate precision (i.e., inter-run variability) to be

171 estimated. In a separate experiment assessing the potential for interactions among

172 complex mixtures of natural compounds, the potency of pure polygodial was compared

173 to that of polygodial enriched with an environmental extract. The extract was produced

174 by eluting $10 \mathrm{~L}$ seawater that $\sim 400 \mathrm{~g}$ of green-lipped mussels, Perna canaliculus

175 Gmelin, had been cultured in for 1 day through a $20-\mathrm{cm}^{3}$ column of Diaion HP20 resin

176 (Mitsubishi Chemical, Tokyo, JP). The column was flushed with $50 \mathrm{~mL}$ ethanol, and the

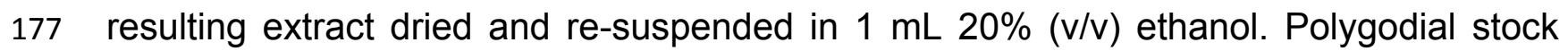

178 solutions prepared in $1 \mathrm{~mL}$ of $20 \%(\mathrm{v} / \mathrm{v})$ ethanol or $1 \mathrm{~mL}$ of extract were added to wells

179 to yield final polygodial concentrations of $2.5,5,10$, and $50 \mathrm{ng} \mathrm{mL}^{-1}(\mathrm{n}=3)$.

\subsection{Data Analyses}


181 Temperature, photoperiod, and substrate data were analyzed using one-way linear

182 mixed-effects modelling (Bolker et al. 2009), with metamorphic progress (Section 2.2)

183 as the response variable, treatment as a fixed factor, and time as a continuous

184 covariate. Replicate was included as a random effect to account for the repeated-

185 measures experimental design. Reported p-values are based on the $t$ distribution of the

186 ratios between the estimates and their standard errors (Pinheiro and Bates 2006).

187 Principal response curves (PRC), a redundancy analysis for multivariate responses in

188 repeated-measures design (Van den Brink and Ter Braak 1998, Van den Brink and Ter

189 Braak 1999), were used with 999 permutations to identify the metamorphic stages that

190 were driving treatment effects ( $>0.5$ signifies strong treatment effect). Mean absolute

191 PRC coefficients for the number of larvae in each metamorphic stage $(A-F)$ at each

192 time point were calculated by averaging the values from the temperature, photoperiod,

193 and substrate datasets. The effects of larval density and vessel size were assessed

194 using a two-way factorial ANOVA with the number of Stage E larvae on day 5 as the

195 response variable. The effect of fill-volume on the number of larvae reaching Stage E

196 within 5 days was tested using a Student's t-test, and carrier solvent using one-way

197 ANOVA followed by Dunnett's post-hoc test. Dose-response curves were plotted for the

198 reference compounds using four parameter logistic curve fitting, and the corresponding

199 concentrations that reduced the number of larvae reaching Stage $E$ by $50 \%$ relative to

200 blank controls $\left(\mathrm{EC}_{50}\right.$ ) calculated (Kuo et al. 1993). Repeatability (relative bias) and

201 intermediate precision for the replicate polygodial experiments were calculated in

202 accordance with USP <1033> (2012). Analyses were performed using R 2.13.1 (R

203 Development Core Team 2015) and SigmaPlot 11.0 (Systat Software Inc. 2015). 
204

205

206 $207 \pm 0.04$ days.

208

209

210

211

212

213

21

215

216

217

218

219

220

221

222

223

224

225

226

\section{Results}

Overall, larvae reached stages A and B of metamorphosis within 1 day, while stage $C$ took $1.2 \pm 0.06$ days, stage $D 3.1 \pm 0.02$ days, stage $E 4.3 \pm 0.03$ days, and stage $F 6.6$

\subsection{Temperature, photoperiod, and substrate}

Larvae responded to variations in temperature, photoperiod, and substrate. Daily metamorphic progress was comparable for larvae reared at 15,18 , or $21^{\circ} \mathrm{C}$ but hindered at $25^{\circ} \mathrm{C}(\mathrm{t}=-4.6, \mathrm{p}<0.001$; Figure $2 \mathrm{~A})$. Metamorphic progress was enhanced relative to the other photoperiod regimes evaluated when exposed to $12: 12 \mathrm{~h}$ subdued light:dark $(t=2.1, p=0.04)$, and slowed by exposure to constant intense light $(t=3.0, p$ $=0.004$; Figure 2B). Metamorphic progress was reduced when the substrate was smooth acrylic $(t=-3.5, p=0.04$; Figure $2 \mathrm{C}$ ) but there were no discernable differences between the other substrate types tested. The statistical differences observed for the temperature $(P R C, F=10.1, p=0.005)$, photoperiod $(P R C, F=8.4, p=0.005)$, and substrate $(P R C, F=1.9, p=0.005)$ datasets were driven by the latter stages of metamorphosis. Mean absolute PRC coefficients revealed a strong treatment response for stages $E(4.1 \pm 0.9)$ and $F(5.1 \pm 1.5)$, compared to weak effects for stages $A$ $(0.0002 \pm 0.0001), B(0.0005 \pm 0.0003), C(0.017 \pm 0.011)$, and $D(0.27 \pm 0.14)$.

\subsection{Larval density, vessel size, and fill volume}

Both density (ANOVA, $F=21.4, p<0.005$ ) and vessel size (ANOVA, $F=13.3, p<$ 0.005) significantly influenced metamorphosis. The proportion of larvae reaching Stage E within 5 days was enhanced above 10 larvae $\mathrm{mL}^{-1}$ for Petri dishes or 5 larvae $\mathrm{mL}^{-1}$ for six- and twelve-well plates. Overall, the greatest proportion of Stage E larvae were 
227 observed in twelve-well plates (Figure 3). Larval metamorphosis was enhanced when

228 wells were filled to capacity ( $t=5.1, p=0.007$ ). In wells filled to capacity, $87 \pm 5 \%$ of

229 larvae completed stage $E$ of metamorphosis within 5 days compared to $45 \pm 5 \%$ in 230 control wells.

\section{$231 \quad 3.3$ Carrier solvent and reference compounds}

232 No detectable effects on larval metamorphosis were observed when carrier solvent was 233 added to the vessels at or below $20 \mu \mathrm{L} \mathrm{mL}^{-1}$ for ethanol or $10 \mu \mathrm{L} \mathrm{mL}^{-1}$ for DMSO. When 234 dosed at $40 \mu \mathrm{L} \mathrm{mL}^{-1}$, the number of larvae that reached stage $E$ was reduced to zero in 235 both cases (ethanol ANOVA: $F=12.6, p<0.005$; Dunnett's $p=0.009$; DMSO ANOVA: $236 \quad F=16.0, p<0.005 ;$ Dunnett's $p=0.02$ ).

237 Polygodial $\left(\mathrm{EC}_{50}=4.5 \mathrm{ng} \mathrm{mL}^{-1}\right)$, portimine $\left(\mathrm{EC}_{50}=1.0 \mathrm{ng} \mathrm{mL}^{-1}\right)$, oroidin $\left(\mathrm{EC}_{50}=\right.$ $\left.2381.1 \mu \mathrm{g} \mathrm{mL}^{-1}\right)$, chlorothalonil $\left(\mathrm{EC}_{50}=0.1 \mu \mathrm{g} \mathrm{mL}-1\right)$, and tolylfluanid $\left(\mathrm{EC}_{50}=0.3 \mu \mathrm{g} \mathrm{mL}^{-1}\right)$ 239 potently inhibited metamorphosis; dose-response curves closely fit the data $\left(R^{2}>0.9\right)$.

240 The polygodial positive controls from the eight independent experiments had negative 241 dose-dependent effects on metamorphosis, with mean reductions in the proportions of 242 larvae reaching stage $E$ varying by $14 \pm 9 \%$ within experiments (i.e., repeatability) and $24317 \pm 3 \%$ between experiments (i.e., intermediate precision; Figure 4, Table 1). However, 244 enrichment with an environmental extract decreased the potency of polygodial (Figure 245 5). The extract alone had no detectable effect on metamorphosis but the observed shift 246 in potency for enriched vs. pure polygodial represents an approximately $80 \%$ increase 247 in $\mathrm{EC}_{50}\left(7.8\right.$ vs. $\left.4.3 \mathrm{ng} \mathrm{mL}^{-1}\right)$. 
248

249 The larvae of

250

25

252

253

254

255

256

257

258

259

260

261

262

263

264

265

266

267

268

269

270

\section{Discussion}

reported previously, they completed metamorphosis to established juveniles inside seven days (Cirino et al. 2002, Hendrickson et al. 2004). The proportion of larvae completing metamorphosis to stage $\mathrm{E}$ within five days exceeded $90 \%$ in some cases, but the performance of the larvae was highly dependent on environmental factors. Temperature and photoperiod strongly influenced metamorphic progress. Although no observable differences were found between 15 and $21^{\circ} \mathrm{C}$, exceeding $21^{\circ} \mathrm{C}$ effectively halted the progression of metamorphosis. A similar optimal temperature range of $12-$ $20^{\circ} \mathrm{C}$ has previously been reported for $C$. savignyi embryogenesis (Nomaguchi et al. 1997). Likewise, the observed enhancement of metamorphic progress for larvae reared under 12:12 $\mathrm{h}$ subdued light:dark fits the general rule that ascidians are initially positively phototactic but switch to being negatively phototactic when competent to settle (McHenry 2005). It follows that free swimming larvae ought to prefer a lighted environment and, when competent to settle, will utilize the dark period to settle and begin metamorphosis.

Compared to temperature and photoperiod, substrate had only limited impact on

C. savignyi larvae. Ascidians are generally considered to be one of the least discerning marine taxa in relation to surface selection (Aldred and Clare 2014), yet there are anecdotal reports of surface selectivity in some species: Ascidia nigra Savigny settlement is negatively correlated with surface wettability (Gerhart et al. 1992), Ascidia interrupta Heller prefer sandblasted surfaces (Rae Flores and Faulkes 2008), and Ascidiella spp. tend to be most abundant on concrete pilings (Andersson et al. 2009). 
271 Many other marine larvae will only attach to surfaces with defined chemical, biological,

272 texture, or wettability characteristics (Pawlik 1992, Wahl 1989). For example, barnacle

273 cyprids prefer smooth surfaces (Berntsson et al. 2000) and Ulva zoospores use quorum

274 sensing signal molecules to seek out suitable biofilm communities (Tait et al. 2005).

275 Granting that only a small number of simple substrate variations were tested and larvae

276 were confined within small vessels, C. savignyi did not display obvious substrate

277 preferences in the experiments performed here. The sides and bottom of the wells were

278 of dissimilar composition, yet larvae adhered indiscriminately to all surfaces. The

279 exception was wells lined with smooth acrylic, where the overall progression of

280 metamorphosis was reduced by approximately $12 \%$ compared to the other surfaces

281 tested. This apparent insensitivity to surface characteristics may contribute to $C$.

282 savignyi's invasive character, whereby larvae will settle on a wide range of available 283 surfaces.

284 When the temperature, photoperiod, and substrate datasets were combined, the 285 earliest developmental stage of metamorphosis to show strong treatment response was 286 stage E when the branchial basket, siphons, and stigmata develop. It has previously 287 been noted that tail-resorption is a particularly sensitive stage of ascidian 288 metamorphosis (e.g., Bishop et al. 2001, Eri et al. 1999, Green et al. 2002) but, in the 289 case of $C$. savignyi, the tail resorption process (Stage B) was little affected by variations 290 in temperature, photoperiod, or substrate. It is not clear why the later stages of 291 metamorphosis were more sensitive to environmental variations but the lecithotrophic 292 nature of $C$. savignyi may be partly responsible. Energy reserves within the larvae may 293 become depleted later in metamorphosis (e.g., Jaeckle 1994, Jaeckle and Manahan 
294 1989, Moran and Manahan 2003), with energy deficient larvae becoming increasing

295 susceptible to sub-optimal environmental conditions.

When larval density and vessel size were subsequently evaluated, the proportion

297 of larvae reaching Stage E of metamorphosis was enhanced above 5 or 10 larvae $\mathrm{mL}^{-1}$, with twelve-well plates having the greatest proportion of Stage E larvae overall. Densitydependent behaviours are common among marine larvae (Hadfield and Paul 2001); examples include the gregariousness of larvae of the barnacle Balanus amphitrite

Darwin (Head et al. 2003), the tubeworm Hydroides dianthus Verrill (Toonen and Pawlik 302 1996), and the oyster Ostrea edulis L. (Bayne 1969). Enhanced metamorphosis in smaller vessels could be a result of conspecific settlement cues or reduced surface area to volume ratio. Under the later scenario, larvae are more likely to contact, and thus adhere to, the internal surfaces of a smaller vessel.

Filling wells to capacity further increased the proportion of Stage E larvae 307 observed after 5 days. When wells were not filled to capacity, some C. savignyi larvae became trapped at the air-water interface and where not considered to have successfully attached (P. Cahill pers. obs.). This phenomenon has been reported previously for other ascidians (e.g., Fletcher and Forrest 2011), and is probably due to

311 the larvae swimming upwards against the force of gravity for the first few hours after 312 hatching (Hendrickson et al. 2004). Because culture waters contact the underside of the 313 lid in wells that were filled to capacity, larvae are provided with an inverted surface upon 314 which to settle and are prevented from them becoming trapped at the air-water 315 interface. In line with these findings, it has been anecdotally observed that $C$. savignyi 
316 adults tend to be congregated on the underside of man-made structures in the sea $(P$.

317 Cahill pers. obs.).

318 Evaluating the effects of select reference compounds on C. savignyi

319 metamorphosis highlighted the potential to use $C$. savignyi larvae to screen for 320 bioactivity under an optimised laboratory setting. The natural antifouling agent 321 polygodial (Cahill and Kuhajek 2014), the algal biotoxin portimine (Selwood et al. 2013), 322 and the synthetic antifouling biocides chlorothalonil and tolyfluanid (Voulvoulis et al. 323 1999) were bioactive in the low $\mathrm{ng} \mathrm{mL}-1$ to high $\mu \mathrm{gL} \mathrm{m}^{-1}$ ranges. Tolerance of $C$. savignyi 324 larvae for the carrier solvents ethanol $\left(20 \mu \mathrm{L} \mathrm{mL}-1\right.$ max.) and DMSO (10 $\left.\mu \mathrm{L} \mathrm{mL}^{-1} \mathrm{max}^{-}\right)$ 325 facilitated screening these compounds, which, with the exception of portimine, have 326 only limited water solubility. The results reported here meet or exceed potency 327 estimates previously determined for these compounds against other organisms. For 328 example, polygodial is effective against fungal pathogens at approximately $1 \mu \mathrm{g} \mathrm{mL}^{-1}$ 329 (Kubo and Himejima 1991), chlorothalonil kills water fleas and fathead minnows at 0.03 $330-0.2 \mu \mathrm{g} \mathrm{mL}^{-1}$ (Sherrard et al. 2002), and tolylfluanid controls seaweed zoospores at $3310.03 \mu \mathrm{g} \mathrm{mL}^{-1}$ (Wendt et al. 2013). However, systems that test the bioactivity of 332 compounds in solution must be carefully interpreted. In nature, marine larvae are 333 typically only exposed to allelopathic metabolites at surfaces, such as when they 334 contact another benthic organisms (Pawlik 1993). Perfusing larval tissues with a 335 compound, as was done here, provides a largely pharmacological rather than ecological 336 context. This pharmacological data is often used as a first step to identify promising 337 bioactive compounds but should always be followed by additional investigations, either 
338 laboratory or field based, where the compound of interest is bound to/released from a

339 surface (Bressy et al. 2014).

When polygodial treatments were included in eight independent experiments

341 performed over a one year period, mean reductions in the proportion of larvae reaching

342 stage $E$ varied by $14 \pm 9 \%$ and $17 \pm 3 \%$ within and between experiments, respectively.

343 These values represent a relatively high degree of consistency for the effects of 344 polygodial exposure, with comparable larval systems returning repeatability estimates in

345 the order of $5-30 \%$ (e.g., Piazza et al. 2012, Ross and Bidwell 2001, Stronkhorst et al.

346 2004). The larvae were unaffected by a complex environmental extract produced from

347 the culture waters of green-lipped mussels but the same extract reduced the potency of 348 polygodial to almost half. This result highlights the potential for changes in potency 349 when working with complex chemical samples, as is common when attempting to 350 isolate natural products (Colegate and Molyneux 2007).

Overall, this study yielded insights into the environmental factors impacting

352 metamorphosis of C. savignyi larvae. This increased understanding of the interplay

353 between the environment and the biology of $C$. savignyi is vital to understanding, and

354 perhaps counteracting, the invasive tendencies of this organism. The findings also

355 provide context for the growing body of research examining the biochemical and genetic

356 determinants of $C$. savignyi metamorphosis. Demonstrating that $C$. savignyi larvae are

357 sensitive to a range of natural and synthetic bioactive agents highlighted one potential

358 application for these larvae. Using C. savignyi larvae to assess the toxicity of

359 compounds under optimised laboratory conditions presents a potentially useful

360 preliminary screening tool but the outcomes of such experiments must be carefully 
361 interpreted and should be followed by ecologically relevant investigations. Nevertheless,

362 chemicals targeting $C$. savignyi metamorphosis could potentially be used to control this 363 pest species.

364 
Aldred N, Clare AS. 2014. Mini-review: Impact and dynamics of surface fouling by solitary and compound ascidians. Biofouling. 30:259-270. Andersson MH, Berggren M, Wilhelmsson D, Öhman MC. 2009. Epibenthic colonization of concrete and steel pilings in a cold-temperate embayment: a field experiment. Helgoland Marine Research. 63:249-260. Bayne B. 1969. The gregarious behaviour of the larvae of Ostrea edulis L. at settlement. Journal of the Marine Biological Association of the United Kingdom. 49:327-356. Berntsson KM, Jonsson PR, Lejhall M, Gatenholm P. 2000. Analysis of behavioural rejection of micro-textured surfaces and implications for recruitment by the barnacle Balanus improvisus. Journal of Experimental Marine Biology and Ecology. 251:59-83.

Bishop CD, Bates WR, Brandhorst BP. 2001. Regulation of metamorphosis in ascidians involves NO\&sol; cGMP signaling and HSP90. Journal of Experimental Zoology. 289:374-384.

Bolker BM, Brooks ME, Clark CJ, Geange SW, Poulsen JR, Stevens MHH, White J-SS. 2009. Generalized linear mixed models: a practical guide for ecology and evolution. Trends in Ecology \& Evolution. 24:127-135. Bressy C, Briand JF, Compère C, Réhel K. 2014. Efficacy testing of biocides and biocidal coatings. In: Biofouling Methods. Wiley Blackwell. p. 332-345. Cahill P, Kuhajek J. 2014. Polygodial: a contact active antifouling biocide. Biofouling. 30:1035-1043.

Cirino P, Toscano A, Caramiello D, Macina A, Miraglia V, Monte A. 2002. Laboratory culture of the ascidian Ciona intestinalis (L.): a model system for molecular developmental biology research. Marine Models Electronic Records. Available at: http://www.mbl.edu/BiologicalBulletin/MMER/cirino/CirTit.htm. Cordell J, Harris L, et al. 1998. A rapid assessment survey of non-indigenous species in the shallow waters of Puget Sound Olympia ,WA.

Colegate SM, Molyneux RJ. 2007. Bioactive natural products: detection, isolation, and structural determination: CRC press.

395 Corbo JC, Di Gregorio A, Levine M. 2001. The ascidian as a model organism in developmental and evolutionary biology. Cell. 106:535-538.

Eri R, Arnold JM, Hinman VF, Green KM, Jones MK, Degnan BM, Lavin MF. 1999. Hemps, a novel EGF-like protein, plays a central role in ascidian metamorphosis. Development. 126:5809-5818.

Fletcher LM, Forrest BM. 2011. Induced spawning and culture techniques for the invasive ascidian Didemnum vexillum (Kott, 2002). Aquatic Invasions. 6:457-464.

Fofonoff PW, Ruiz GM, Steves B, Carlton JT. 2003. Ciona savignyi. National Exotic Marine and Estuarine Species Information System. 18-Jul-2014; Available at: http://invasions.si.edu/nemesis/browseDB/SpeciesSummary.jsp?TSN=-122.

Gerhart D, Rittschof D, Hooper I, Eisenman K, Meyer A, Baier R, Young C. 1992. Rapid and inexpensive quantification of the combined polar components of surface wettability: application to biofouling. Biofouling. 5:251-259. 
408 Green K, Russell B, Clark R, Jones M, Garson M, Skilleter G, Degnan B. 2002. A 409 sponge allelochemical induces ascidian settlement but inhibits metamorphosis. Marine 410 Biology. 140:355-363.

411 Hadfield MG, Paul VJ. 2001. Natural chemical cues for settlement and metamorphosis 412 of marine invertebrate larvae. In: CRC Press, Boca Raton, FL. p. 431-461.

413 Head R, Overbeke K, Klijnstra J, Biersteker R, Thomason J. 2003. The effect of 414 gregariousness in cyprid settlement assays. Biofouling. 19:269-278.

415 Hendrickson C, Christiaen L, Deschet K, Jiang D, Joly J-S, Legendre L, Nakatani Y, 416 Tresser J, Smith WC. 2004. Culture of adult ascidians and ascidian genetics. Methods 417 in Cell Biology. 74:143-170.

418 Imai KS, Satoh N, Satou Y. 2002a. Early embryonic expression of FGF4/6/9 gene and 419 its role in the induction of mesenchyme and notochord in Ciona savignyi embryos. 420 Development. 129:1729-1738.

421 Imai KS, Satou Y, Satoh N. 2002b. Multiple functions of a Zic-like gene in the embryos. Development. 129:2723-2732.

Jackson D, Leys SP, Hinman VF, Woods R, Lavin MF, Degnan BM. 2002. Ecological regulation of development: induction of marine invertebrate metamorphosis. International Journal of Developmental Biology. 46:679-686.

Jaeckle W. 1994. Rates of energy consumption and acquisition by lecithotrophic larvae of Bugula neritina (Bryozoa: Cheilostomata). Marine Biology. 119:517-523.

Jaeckle WB, Manahan DT. 1989. Growth and energy imbalance during the development of a lecithotrophic molluscan larva (Haliotis rufescens). The Biological Bulletin. 177:237-246.

Jiang D, Smith W. 2005. Self-and cross-fertilization in the solitary ascidian Ciona savignyi. The Biological Bulletin. 209:107-112.

Kimura Y, Yoshida M, Morisawa M. 2003. Interaction between noradrenaline or adrenaline and the $\beta$ 1-adrenergic receptor in the nervous system triggers early metamorphosis of larvae in the ascidian, Ciona savignyi. Developmental Biology. 258:129-140.

Kourakis M, Newman-Smith E, Smith W. 2010. Key steps in the morphogenesis of a cranial placode in an invertebrate chordate, the tunicate Ciona savignyi. Developmental Biology. 340:134-144.

Kubo I, Himejima M. 1991. Anethole, a synergist of polygodial against filamentous microorganisms. Journal of Agricultural and Food Chemistry. 39:2290-2292.

Kuo J, Mitchell D, Tuerke T. 1993. SigmaPlot: Scientific graph system. Transforms and curve fitting reference. Corte Madera: Jandel Scientific.

Lambert CC, Lambert G. 1998. Non-indigenous ascidians in southern California harbors and marinas. Marine Biology. 130:675-688.

Lemaire P. 2011. Evolutionary crossroads in developmental biology: the tunicates. Development. 138:2143-2152.

McHenry MJ. 2005. The morphology, behavior, and biomechanics of swimming in ascidian larvae. Canadian Journal of Zoology. 83:62-74.

Moran AL, Manahan DT. 2003. Energy metabolism during larval development of green and white abalone, Haliotis fulgens and H. sorenseni. The Biological Bulletin. 204:270277. 
454 Morse DE. 1990. Recent progress in larval settlement and metamorphosis: closing the 455 gaps between molecular biology and ecology. Bulletin of Marine Science. 46:465-483.

456 Nomaguchi TA, Nishijima C, Minowa S, Hashimoto M, Haraguchi C, Amemiya S, 457 Fujisawa H. 1997. Embryonic thermosensitivity of the ascidian, Ciona savignyi. 458 Zoological Science. 14:511-515.

459 Pawlik JR. 1992. Chemical ecology of the settlement of benthic marine invertebrates. Oceanography and Marine Biology: An Annual Review. 30:273-335.

461

462

463 Pawlik JR. 1993. Marine invertebrate chemical defenses. Chemical Reviews. 93:19111922.

Piazza V, Ferioli A, Giacco E, Melchiorre N, Valenti A, Del Prete F, Biandolino F, Dentone L, Frisenda P, Faimali M. 2012. A standardization of Amphibalanus (Balanus) amphitrite (Crustacea, Cirripedia) larval bioassay for ecotoxicological studies. Ecotoxicology and Environmental Safety. 79:134-138.

467 Pinheiro J, Bates D. 2006. Mixed-effects models in S and S-PLUS NJ, USA: Springer Science \& Business Media.

469 R Development Core Team. 2015. R: A language and environment for statistical computing. R Foundation for Statistical Computing. http://www.R-project.org/. Rae Flores A, Faulkes Z. 2008. Texture preferences of ascidian tadpole larvae during settlement. Marine and Freshwater Behaviour and Physiology. 41:155-159. Rodriguez SR, Ojeda FP, Inestrosa NC. 1993. Settlement of benthic marine invertebrates. Marine Ecology Progress Series. 97:193-207. Ross K, Bidwell J. 2001. A 48-h larval development toxicity test using the marine polychaete Galeolaria caespitosa Lamarck (Fam. Serpulidae). Archives of Environmental Contamination and Toxicology. 40:489-496. Sasakura Y, Mita K, Ogura Y, Horie T. 2012. Ascidians as excellent chordate models for studying the development of the nervous system during embryogenesis and metamorphosis. Development, Growth \& Differentiation. 54:420-437. Satoh N. 2003. The ascidian tadpole larva: comparative molecular development and genomics. Nature Reviews Genetics. 4:285-295. Satoh N, Levine M. 2005. Surfing with the tunicates into the post-genome era. Genes \& Development. 19:2407-2411. Schubert M, Escriva H, Xavier-Neto J, Laudet V. 2006. Amphioxus and tunicates as evolutionary model systems. Trends in Ecology \& Evolution. 21:269-277.

Selwood Al, Wilkins AL, Munday R, Shi F, Rhodes LL, Holland PT. 2013. Portimine: a bioactive metabolite from the benthic dinoflagellate Vulcanodinium rugosum. Tetrahedron Letters. 54:4705-4707.

Sherrard R, Murray-Gulde C, Rodgers Jr J, Shah Y. 2002. Comparative toxicity of chlorothalonil and chlorpyrifos: Ceriodaphnia dubia and Pimephales promelas. Environmental Toxicology. 17:503-512.

Smith KF, Cahill PL, Fidler AE. 2010. First record of the solitary ascidian Ciona savignyi Herdman, 1882 in the Southern Hemisphere. Aquatic Invasions. 5:363-368.

Stronkhorst J, Ciarelli S, Schipper C, Postma J, Dubbeldam M, Vangheluwe M, Brils J, Hooftman R. 2004. Inter-laboratory comparison of five marine bioassays for evaluating the toxicity of dredged material. Aquatic Ecosystem Health \& Management. 7:147-159. SigmaPlot. 2015. San Jose, California: Systat Software Inc. 
499 Tait K, Joint I, Daykin M, Milton DL, Williams P, Camara M. 2005. Disruption of quorum 500 sensing in seawater abolishes attraction of zoospores of the green alga Ulva to bacterial 501 biofilms. Environmental Microbiology. 7:229-240.

502 Toonen RJ, Pawlik J. 1996. Settlement of the tube worm Hydroides dianthus 503 (Polychaeta: Serpulidae): cues for gregarious settlement. Marine Biology. 126:725-733.

504 Tsukamoto S, Kato H, Hirota H, Fusetani N. 1996. Mauritiamine, a new antifouling 505 oroidin dimer from the marine sponge Agelas mauritiana. Journal of Natural Products. 506 59:501-503.

507 USP <1033>. 2012. Biological Assay Validation. In: United States Pharmocopeia. p. 25. 508 Van den Brink PJ, Ter Braak CJ. 1998. Multivariate analysis of stress in experimental 509 ecosystems by principal response curves and similarity analysis. Aquatic Ecology. 510 32:163-178.

511 Van den Brink PJ, Ter Braak CJ. 1999. Principal response curves: Analysis of 512 time-dependent multivariate responses of biological community to stress. Environmental 513 Toxicology and Chemistry. 18:138-148.

514 Voulvoulis N, Scrimshaw M, Lester J. 1999. Alternative antifouling biocides. Applied 515 Organometallic Chemistry. 13:135-143.

516 Wahl M. 1989. Marine epibiosis. I. Fouling and antifouling: some basic aspects. Marine 517 Ecology Progress Series. 58:175-189.

518 Wendt I, Arrhenius Å, Backhaus T, Hilvarsson A, Holm K, Langford K, Tunovic T, 519 Blanck H. 2013. Effects of five antifouling biocides on settlement and growth of 520 zoospores from the marine macroalga Ulva lactuca L. Bulletin of Environmental 521 Contamination and Toxicology. 91:426-432.

522 Zvyagintsev AY, Sanamyan K, Kashenko S. 2007. On the introduction of the ascidian 523 Ciona savignyi Herdman, 1882 into Peter the Great Bay, Sea of Japan. Russian Journal 524 of Marine Biology. 33:133-136. 
525 Figure 1. Stages of Ciona savignyi larval development. At the moment of settlement,

526

527

528

529

530

531

532

533

534

535

536

537

538

539

540

541

542 metamorphosis is triggered (Stage A). Tail resorption is completed in a short time (Stage B). The outer tunic layer is cut off (Stages $C \& D$ ), and then two lateral siphons and a pair of functional stigmata develop (Stage E). The number of stigmata on each side of the branchial basket increases (Stage F), completing metamorphosis. Scale bars $=300 \mu \mathrm{m}$.

Figure 2. Effects of $(A)$ temperature, $(B)$ photoperiod, and (C) substrate on Ciona savignyi larvae. Metamorphic progress was calculated based on the sum of the number of individuals reaching each metamorphic stage converted to numerals $(A=1, B=2, C=3, D=4, E=5, F=6)$. Values are means $(n=3) \pm 95 \%$ confidence interval.

Figure 3. Proportion of Ciona savignyi larvae completing Stage E of metamorphosis within 5 days for various combinations of larval density and vessel size. Values are means $(n=3) \pm 95 \%$ confidence interval.

Figure 4. Relative bias from eight independent experiments for the effects of polygodial treatments on Ciona savignyi metamorphosis. Values are means $(n=8)$ relative to a known dose-response relationship for polygodial $\pm 95 \%$ confidence interval.

Figure 5. Relative bias for the effects of polygodial enriched with an environmental extract on Ciona savignyi metamorphosis. Values are means $(n=3)$ relative to pure polygodial $\pm 95 \%$ confidence interval. 
545 Table 1. Variance component estimates and overall variability for the effects of polygodial treatments on Ciona savignyi metamorphosis. 


\section{1}

Stages of Ciona savignyi larval development.

Stages of Ciona savignyi larval development. At the moment of settlement, metamorphosis is triggered (Stage A). Tail resorption is completed in a short time (Stage B). The outer tunic layer is cut off (Stages $C \& D$ ), and then two lateral siphons and a pair of functional stigmata develop (Stage E). The number of stigmata on each side of the branchial basket increases (Stage F), completing metamorphosis. Scale bars $=300 \mu \mathrm{m}$. 


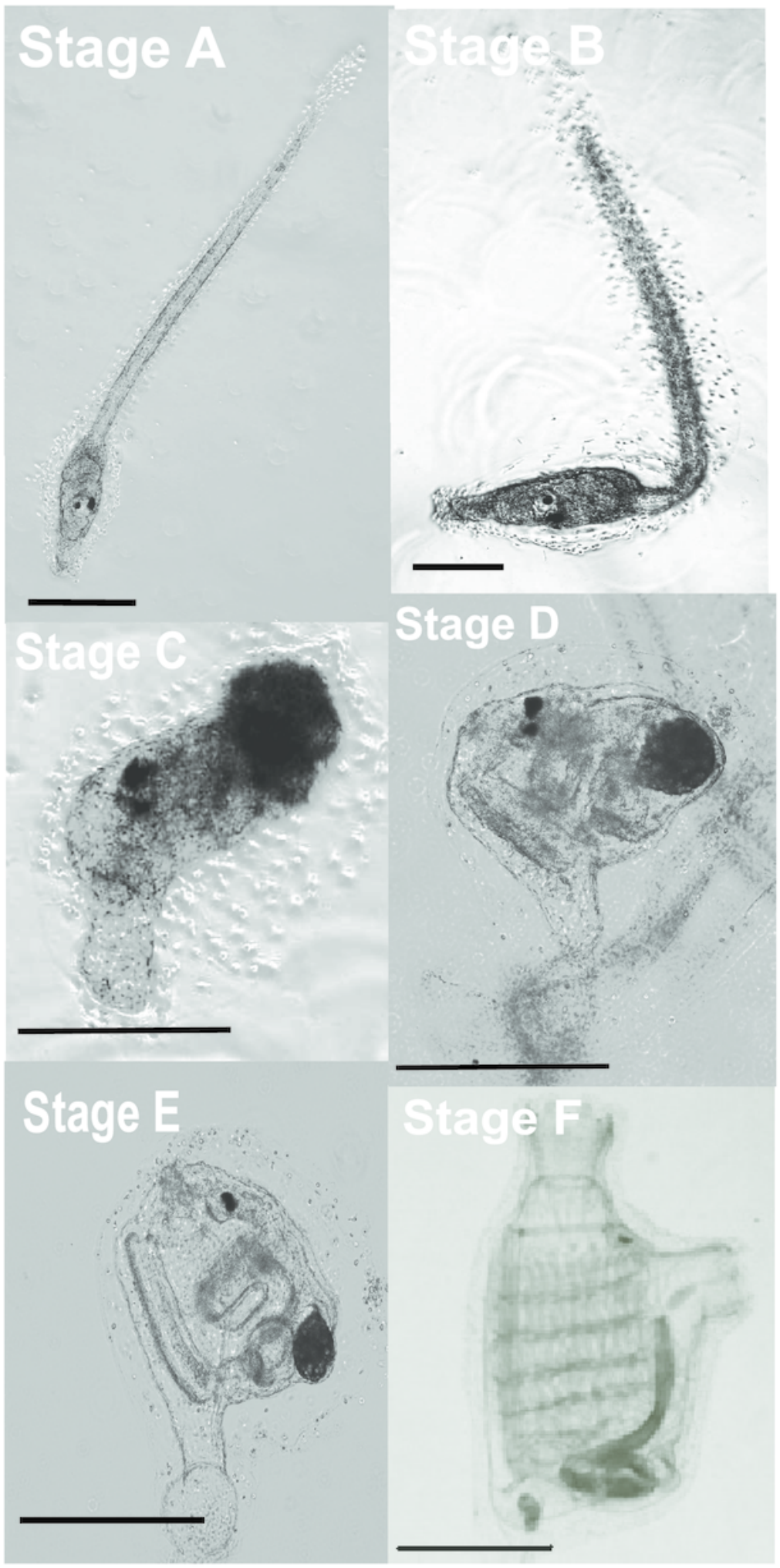


2

Effects of (A) temperature, (B) photoperiod, and (C) substrate on Ciona savignyi larvae.

Effects of (A) temperature, (B) photoperiod, and (C) substrate on Ciona savignyi larvae.

Metamorphic progress was calculated based on the sum of the number of individuals

reaching each metamorphic stage converted to numerals $(A=1, B=2, C=3, D=4, E=5$, $F=6)$. Values are means $(n=3) \pm 95 \%$ confidence interval. 


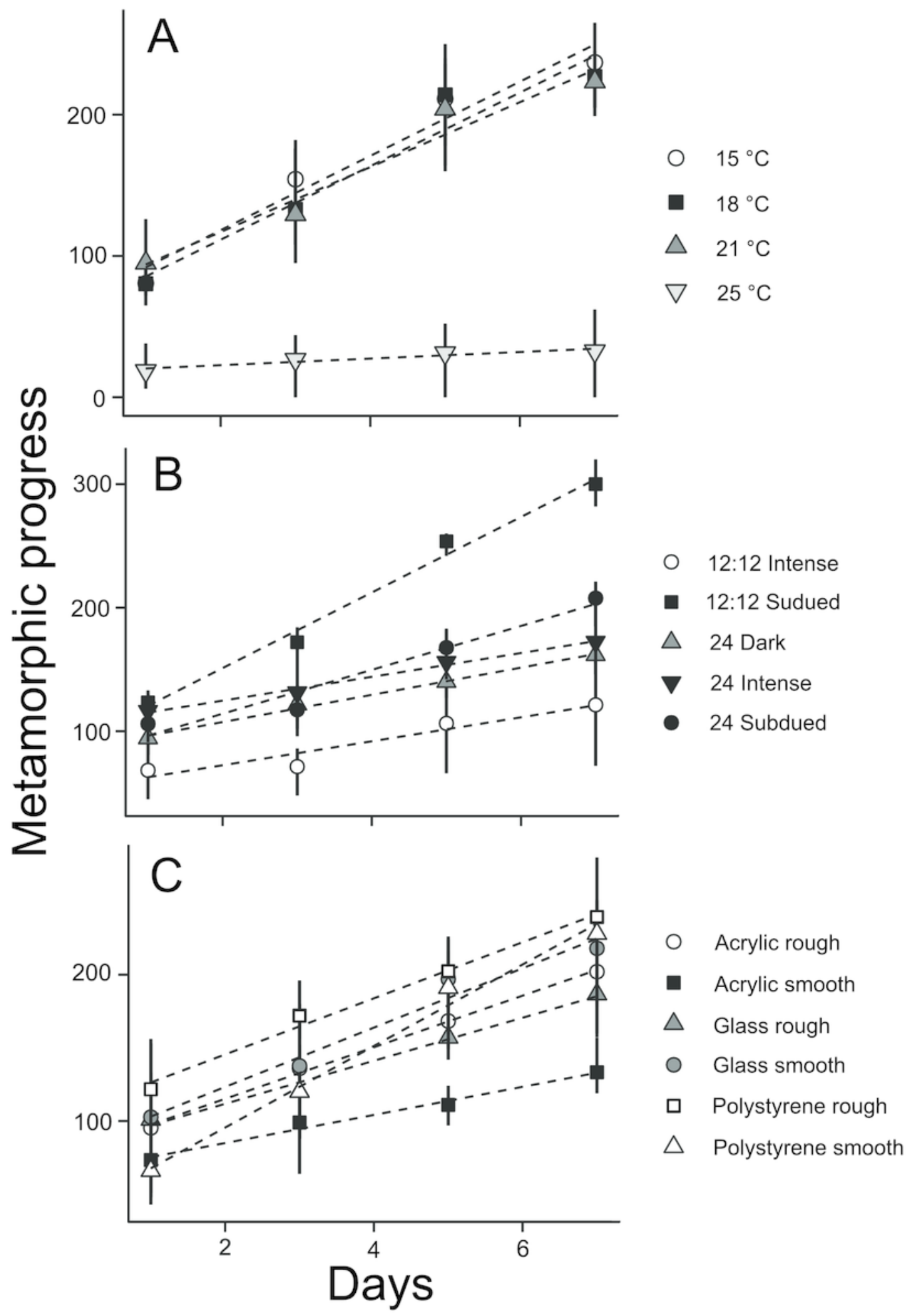


3

Proportion of Ciona savignyi larvae completing metamorphosis

Proportion of Ciona savignyi larvae completing Stage E of metamorphosis within 5 days for various combinations of larval density and vessel size. Values are means $(n=3) \pm 95 \%$ confidence interval.

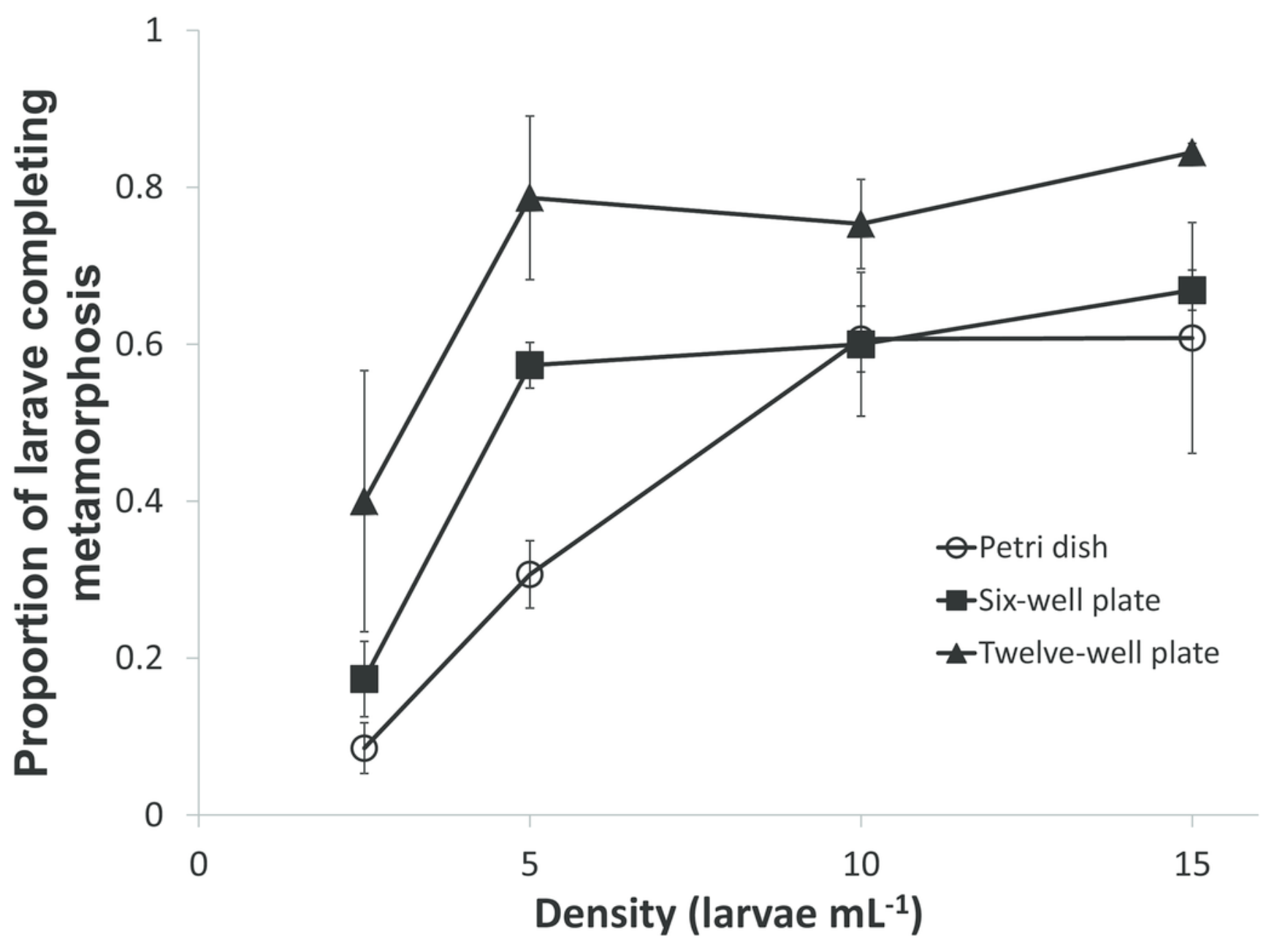


4

Relative bias for polygodial treatments.

Relative bias from eight independent experiments for the effects of for polygodial treatments on Ciona savignyi metamorphosis. Values are means $(n=8)$ relative to a known doseresponse relationship for polygodial $\pm 95 \%$ confidence interval.

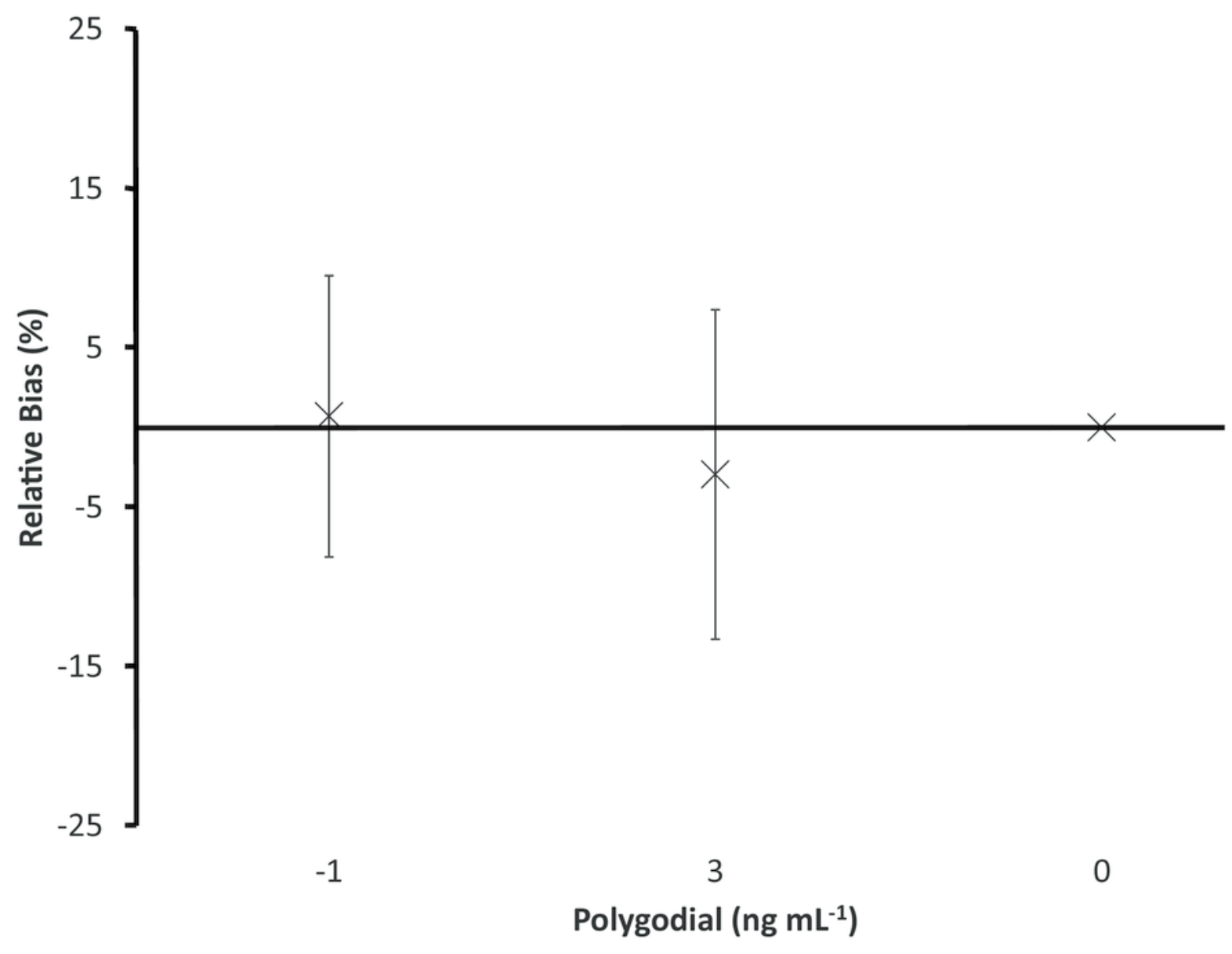




\section{5}

Relative bias for polygodial enriched with an environmental extract.

Relative bias for the effects of polygodial enriched with an environmental extract on Ciona savignyi metamorphosis. Values are means $(n=3)$ relative to pure polygodial $\pm 95 \%$ confidence interval.

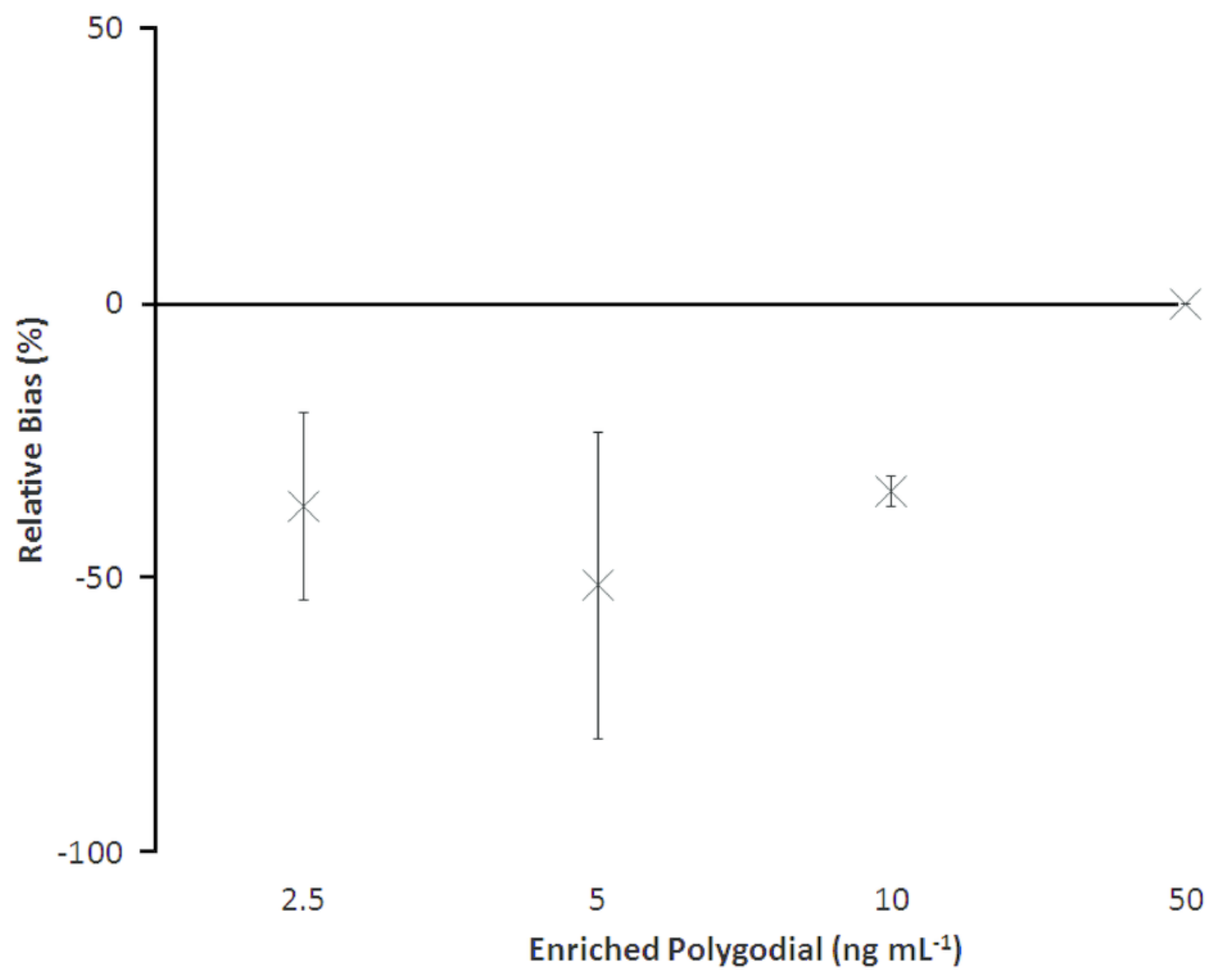


Table $\mathbf{1}$ (on next page)

Overall variability for polygodial treatments. 
1 Table 1. Variance component estimates and overall variability for the effects of polygodial treatments on Ciona savignyi metamorphosis.

\begin{tabular}{|c|c|c|c|}
\hline & $5 \mathrm{ng} \mathrm{mL}^{-1}$ & $10 \mathrm{ng} \mathrm{mL}^{-1}$ & Mean \\
\hline un) & -0.013 & 0.002 & -0.005 \\
\hline Var (Error) & 0.033 & 0.030 & 0.031 \\
\hline Overall & $15 \%$ & $20 \%$ & $17 \%$ \\
\hline
\end{tabular}

